\title{
Evaluation of Mechanical Strength Characteristics of Double Ducted Unmanned Amphibious Aerial Vehicle using Finite Element Analysis
}

\author{
Gokul Raj P..$^{a^{*}}$, Balasubramanian Esakki ${ }^{b}$ \\ a,b Department of Mechanical Engineering \\ Vel Tech Rangarajan Dr. Sagunthala R \& D Institute of Science and Technology, Chennai, India \\ E-mails: agokulrajprithiv@gmail.com, besak.bala@gmail.com \\ ${ }^{*}$ Corresponding author \\ Surendar Ganesan \\ Department of Aeronautical Engineering \\ Vel Tech Rangarajan Dr. Sagunthala R \& D Institute of Science and Technology, Chennai, India \\ E-mail: suren.ganesh.2007@gmail.com
}

(Received November 19, 2018; January 24, 2019)

\begin{abstract}
Unmanned Aerial Vehicles are extensively exploited for diverse applications importantly surveillance, defence and military, photography. Development of unmanned amphibious vehicle with integrating features of hovercraft principles and multirotor to navigate along and above the water surface, land surface and flying in the air is challenging demand. This article presents conceptual design of amphibious vehicle for the payload capacity of $7 \mathrm{~kg}$ with an endurance of 20 minutes and provision for mounting water sampler to collect water samples in remote water bodies. Structural strength characteristics of each part of the amphibious vehicle and integrity of same are analysed by Finite Element Analysis. FEA results indicated that the designed amphibious vehicle structure is well within the stress limit and minimal displacement is obtained. Based on structural analysis materials for various parts of the amphibious vehicle are determined and integrated structure is analysed.
\end{abstract}

Keywords- Amphibious UAV, Hovercraft, Quadcopter, Structural analysis, Finite element analysis.

\section{Introduction}

Unmanned Aerial Vehicles (UAVs) are classified with reference to body shape and size are fixed, flapping and rotary wings (Valavanis and Vachtsevanos, 2015). These UAVs made vivid impact in multifaceted applications including environmental monitoring, search and rescue, bridge inspection, traffic monitoring, mapping of mines, wild life surveying and precision agriculture etc (Hassanalian and Abdelkefi, 2017). Design (Yayla et al., 2013) and development of aerial vehicles (Collins, 1993; Pisanich and Morris, 2002) with diverse pay load capacity (Hasnan and Wahab, 2012) and an ability to perform necessary missions pertaining to specific applications are in the rise. There are few types of research works (Frejek and Nokleby, 2018) on the development of amphibious vehicles (Detweiler et al. 2012), the design of hover craft systems (Amyot, 1989) and many works have been carried out on the development of quad rotor systems (Harkins et al., 2005). However, usage of UAVs in water quality monitoring and collection of water samples in remote water bodies are scarce. Especially, the design of amphibian characteristics UAVs which can fly, land and glide along the water surface imposing lot of challenges in terms of control in flight transition, selection of materials, propulsion, energy consumption and pay load capacity. In addition, other factors such as durability, reliability, safety and minimal cost are utmost important for industrial demand and customer requirement (Liu et al., 2018). There are few floating UAVs, which have been developed and commercialized in the market. However, integrating the 
International Journal of Mathematical, Engineering and Management Sciences

Vol. 4, No. 2, 420-431, 2019

https://dx.doi.org/10.33889/IJMEMS.2019.4.2-034

characteristics of the multi rotor and hover craft systems are not being explored in the literature. These vehicles are aimed to cover large areas of water bodies in short span of time. Unlike other floating vehicles, due to the principle of hovercraft, the friction between the vehicle and water surface is avoided and there by a considerable amount of energy can be saved. (Kumar et al., 2018) The vertical take-off and landing ability of vehicle can position the vehicle in precise water locations across rivers, ponds and other water bodies to collect water samples. Design of amphibious vehicle with sufficient strength characteristics (Gunwant, 2019) to withstand lift conditions and carrying necessary payload are considered to be challenging (Solly et al., 2018). In this work, an amphibious conceptual model is developed to carry a pay load of $7 \mathrm{~kg}$. Finite element analysis is performed for UAV frame, hull, skirt and amphibious structure through varying the materials to examine the structural strength and integrity of the structure (Vinayaga et al., 2018). Modal analysis is carried out to determine the natural frequency of the amphibious structure. A scaled down model is constructed through integrating the sub systems of quad rotor and hovercraft.

\section{Conceptual Model of Amphibious Vehicle}

Amphibious model is conceptualized through integrating the principles of Quadcopter and Hovercraft as shown in Figure 1. The concept is a hybrid prototype of two different vehicles. The basic $\mathrm{H}$ configuration - UAV frame is used as the prototype design. For the vehicle to be airborne, eight brushless electric motors and propellers are assembled to generate sufficient thrust force to lift the entire vehicle. A water sampler mechanism is assembled at the centre of the vehicle. For surface missions and tasks, the prototype can be transformed into a hovercraft. It has two electronic duct fans (EDFs) mounted on the hull that inflates the skirt and another EDF is positioned at the rear to achieve directional control while in hovercraft mode which acts as a rudder. The batteries, electronic accessories, flight controllers and other electrical elements are appropriately distributed to balance the centre of gravity of the vehicle.

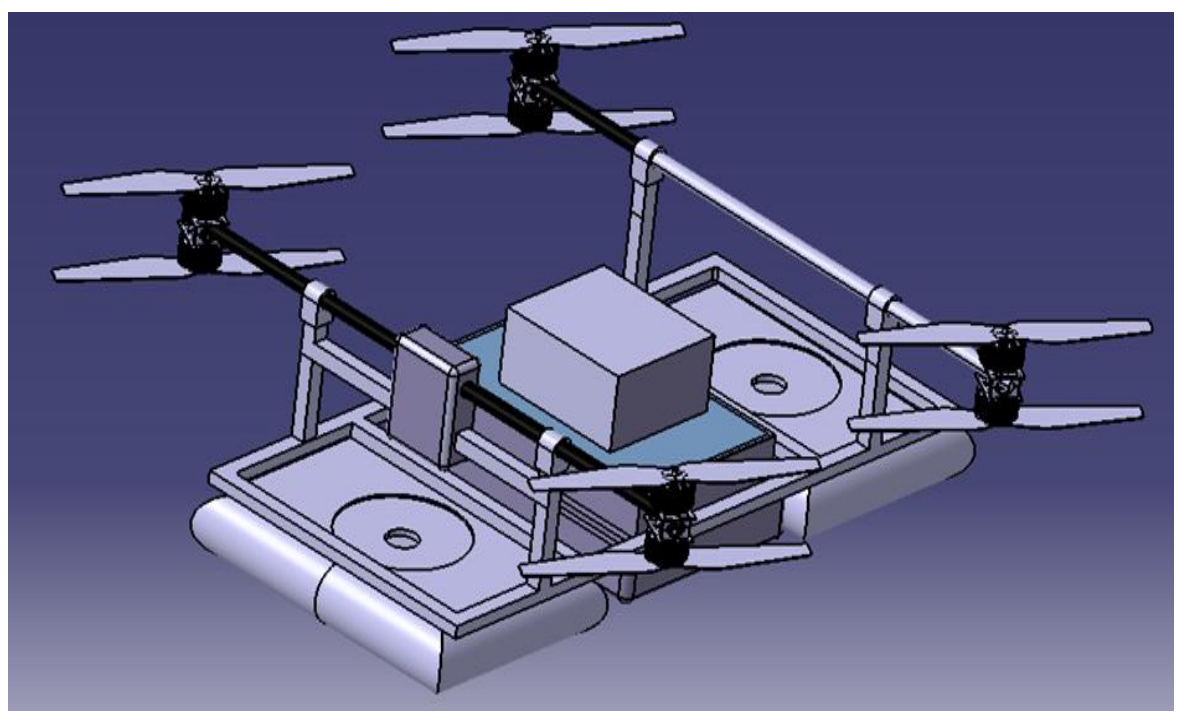

Figure 1. Conceptualized model of amphibious vehicle 
International Journal of Mathematical, Engineering and Management Sciences

Vol. 4, No. 2, 420-431, 2019

https://dx.doi.org/10.33889/IJMEMS.2019.4.2-034

\section{Structural analysis of amphibious UAV}

To identify suitable material for each amphibious part to withstand thrust forces, payload and other load carrying members, structural analysis is performed through considering various materials. They are selected with respect to several criteria including strength to weight ratio, manufacturability, cost and also the availability of materials.

\subsection{Quad Rotor Frame}

The quad rotor frame is meshed (Figure 2) using Mechanical mesh. Finite element (FE) model consists of 11329 nodes and 4269 elements. As for the boundary conditions (Figure 3), the generation of thrust force from each propeller of about $125 \mathrm{~N}$ is applied at each corner of the motor frame, central load of $7 \mathrm{~kg}$ as a payload is concentrated, batteries and other loads are distributed appropriately in the frame structure. For various materials, structural analysis is performed to determine the minimal displacement and stress and their results are given in Table 1. Carbon fibre attained minimal displacement and stress than other materials. However, based on ease of availability, aluminum is chosen for the present study which is carbon fibre. FEA results of aluminium having maximum stress of $11.18 \mathrm{MPa}$ (Figure 4) and displacement of $0.96 \mathrm{~mm}$ (Figure 5) suggested that, aluminium can withstand the thrust force and other distributed loads. It is less weight, better corrosion resistance and high strength to weight ratio.

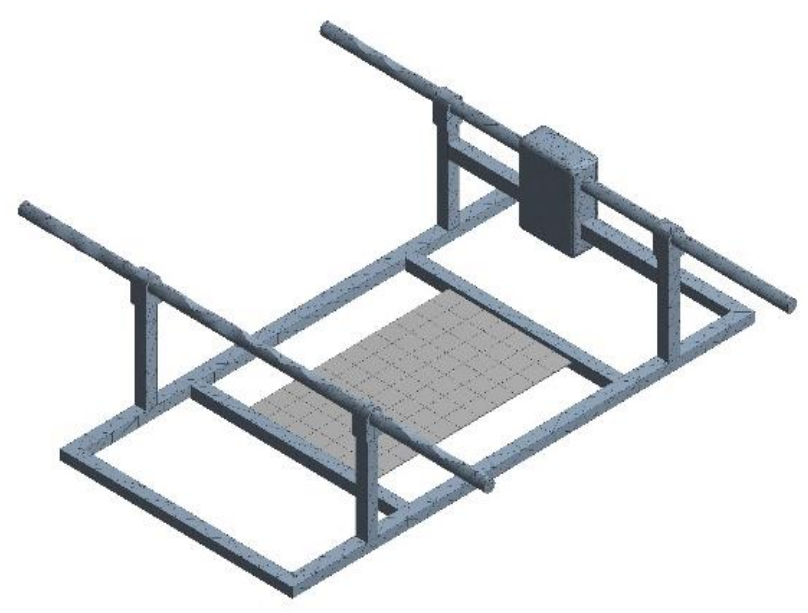

Figure 2. Frame with mesh 
International Journal of Mathematical, Engineering and Management Sciences

Vol. 4, No. 2, 420-431, 2019

https://dx.doi.org/10.33889/IJMEMS.2019.4.2-034

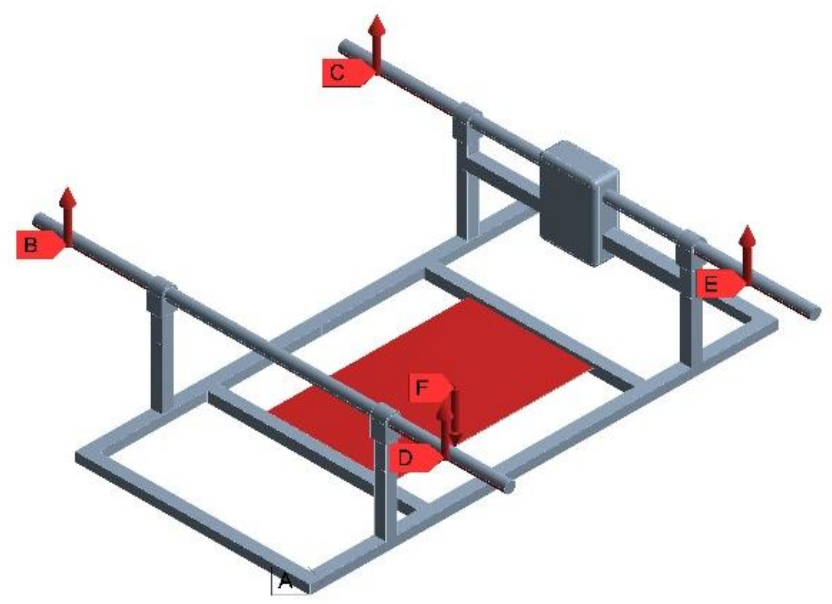

Figure 3. Frame with boundary condition

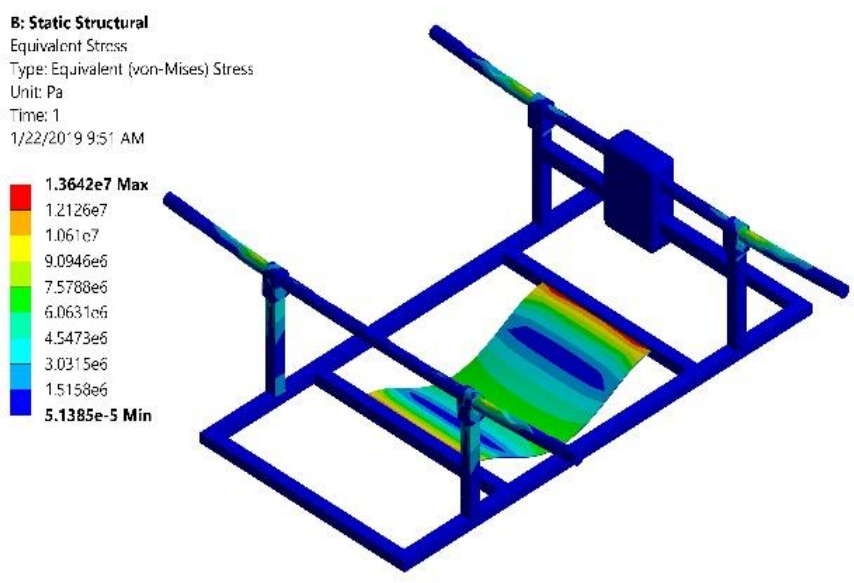

Figure 4. Stress contour of UAV frame

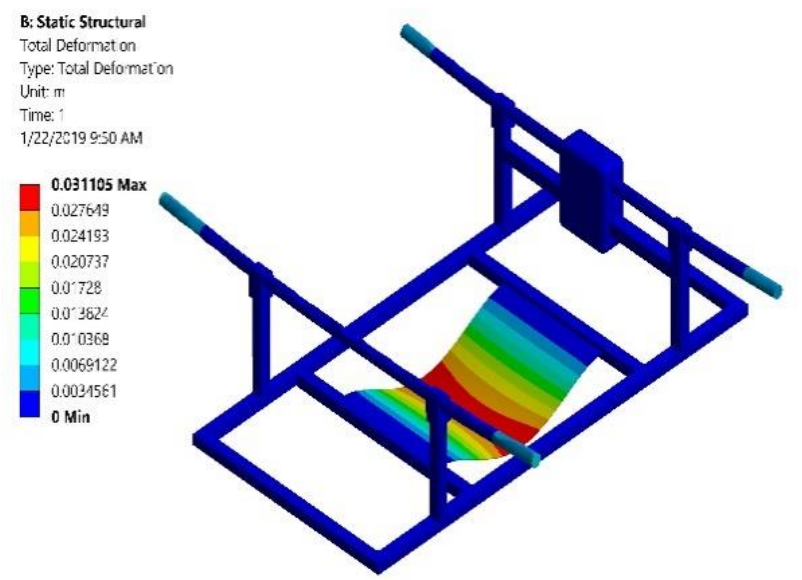

Figure 5. Deformation of UAV frame 
International Journal of Mathematical, Engineering and Management Sciences

Vol. 4, No. 2, 420-431, 2019

https://dx.doi.org/10.33889/IJMEMS.2019.4.2-034

Table 1. Structural analysis results of various materials for UAV frame

\begin{tabular}{|l|c|c|c|}
\hline Materials & Max stress (MPa) & Max displacement (mm) & Structure mass (kg) \\
\hline Aluminium & 11.18 & 0.96 & 14.23 \\
\hline Carbon fiber & 11.16 & 0.29 & 9.247 \\
\hline ABS & 11.20 & 26.02 & 5.445 \\
\hline PEEK & 11.23 & 17.81 & 6.678 \\
\hline ULTEM & 11.16 & 23.83 & 6.524 \\
\hline
\end{tabular}

Polymers such as ABS, PEEK and ULTEM are light weight but may not be the best choice as they do not provide sufficient stiffness. Thus, with respect to cost and availability criteria, aluminum is preferred over carbon fiber.

\subsection{Hull}

The hull serves as a platform to fasten the frame of the quadrotor together and hold the electronics hub. It also incorporates all hardware units, propulsion, wireless communications, soldered circuitry, and battery. The structure is quite particular as it is made up of anisotropic materials. The panel made of wooden sheet and foam core and are sandwiched together to achieve minimal weight without compromising structure strength. The FEA is performed for a differential pressure of $1500 \mathrm{~Pa}$ acting on the internal plenum chamber. The pressure load is due to the air intake by the two EDFs mounted on the hull which inflate the skirt when the vehicle is performing manoeuvres in hovercraft mode. The FE model consists of 9633 nodes and 4789 elements (Figure 6). It has a good mesh quality with an average aspect ratio of 2.64. The boundary conditions (Figure 7) including $10 \mathrm{~kg}$ battery weight and other loads of $1.0 \mathrm{~kg}$ acting on the hull is also considered for the analysis. Simulation results indicated that, maximum stress (Figure 8) and deformation (Figure 9).

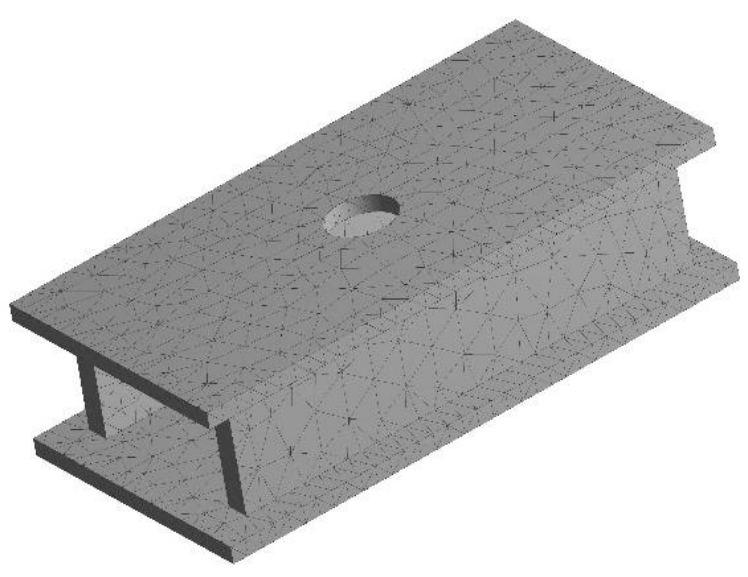

Figure 6. Hull model with mesh 
International Journal of Mathematical, Engineering and Management Sciences

Vol. 4, No. 2, 420-431, 2019

https://dx.doi.org/10.33889/IJMEMS.2019.4.2-034

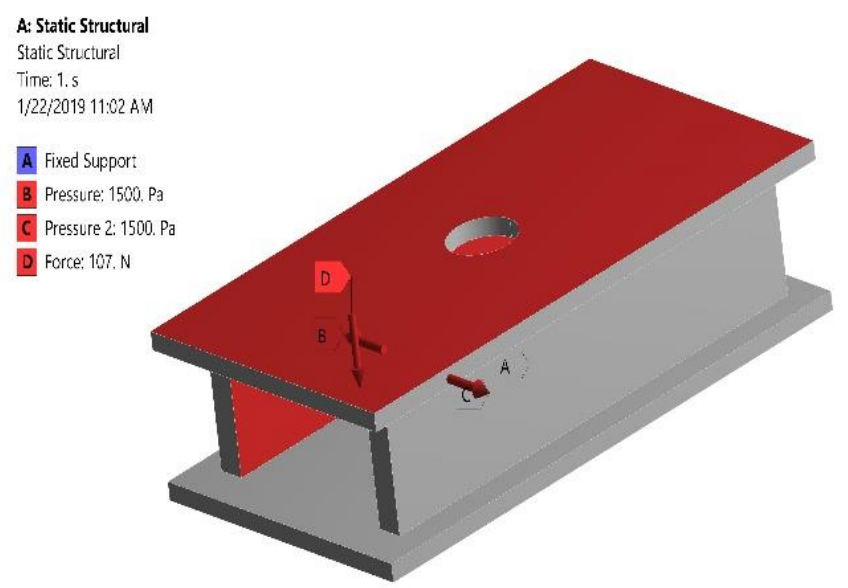

Figure 7. Hull model with boundary conditions

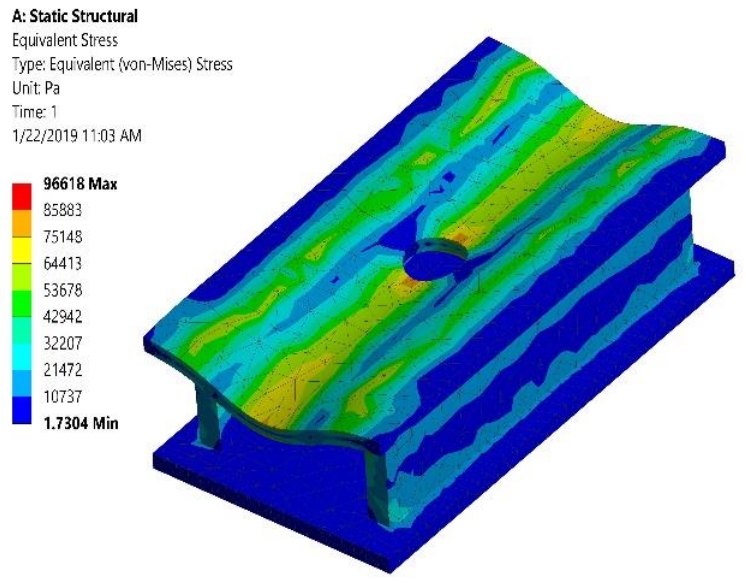

Figure 8. Stress contour of hull

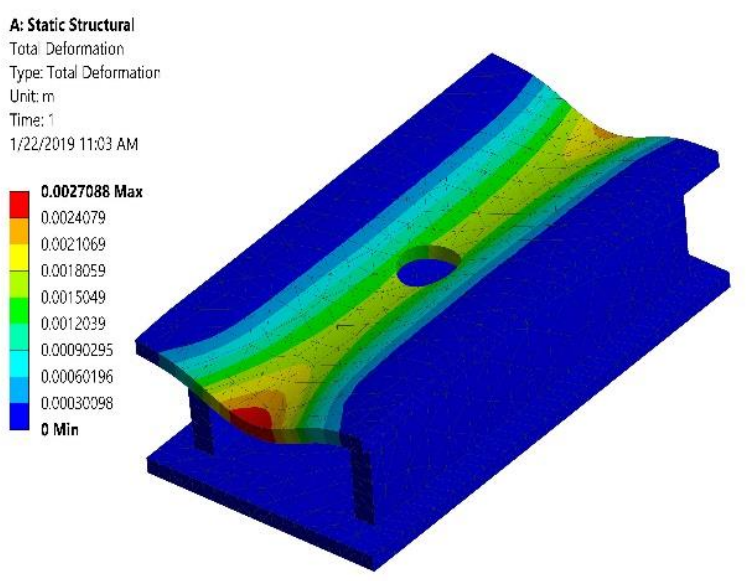

Figure 9. Deformation of hull 
International Journal of Mathematical, Engineering and Management Sciences

Vol. 4, No. 2, 420-431, 2019

https://dx.doi.org/10.33889/IJMEMS.2019.4.2-034

The hull material made of wood / foam sandwich structure has a good strength to weight ratio. The wood / foam core panel is capable of withstanding the payload, battery weight and other electronic modules without exceeding its yield strength and breaking limit.

\subsection{Skirt}

Amongst hovercraft skirt design, the bag skirt approach is unique in which the pressurized airflow from the blower is split among two independent portions: air cushion beneath the craft, which provides lift; and the enclosed volume contained by the bag skirt. Instinctively, the pressure in the skirt must be greater than or equal than the pressure in the air cushion. The pressure differential between the two portions directly influences hovercraft performance, with a trade-off between stability and uneven-surface performance. The skirt is only inflated during hovercraft mode and it creates cushioning effect between the vehicle and water surface. Supposing there is no pressure loss in the plenum chamber, the same internal pressure of $1500 \mathrm{~Pa}$ is applied along the periphery of the structure (Figure 11) and static analysis is performed for diverse materials as given in Table 2. In this case, the thickness of the skirt is almost negligible compared to the size of the component and therefore 2D triangular element is used to keep aspect ratio closer to 1 . The skirt finite element model (Figure 10) is of 29954 nodes and 16450 elements. The FE model has obtained an excellent mesh quality with an average aspect ratio of 0.524 .

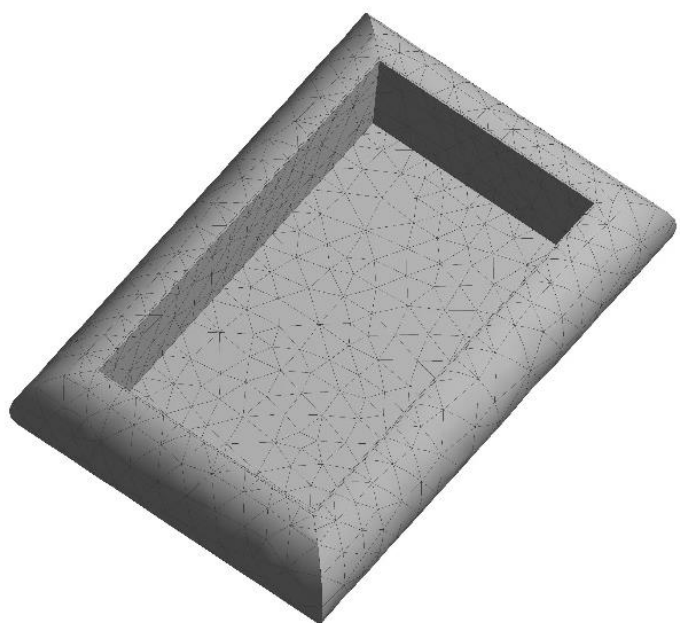

Figure 10. FE skirt model with mesh

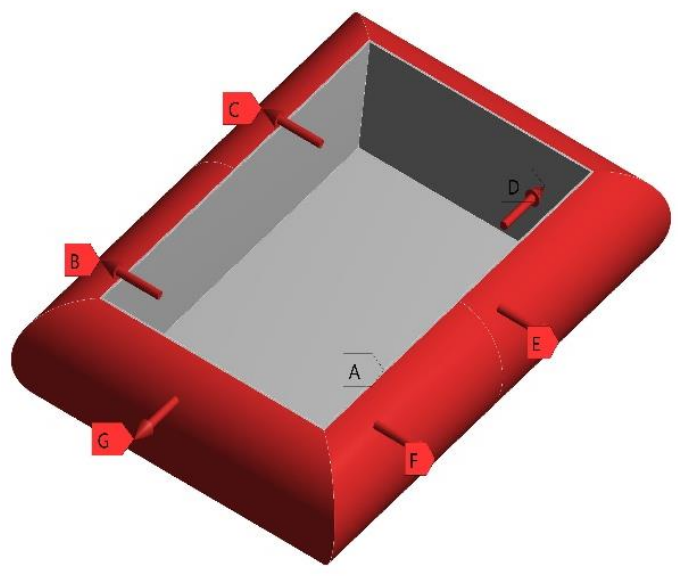

Figure 11. FE skirt with boundary conditions

Table 2. Structural analysis results for various skirt materials

\begin{tabular}{|l|c|c|c|}
\hline Materials & Max stress (MPa) & Max displacement (mm) & Structure mass (kg) \\
\hline Natural rubber & 0.78 & 6.95 & 5.62 \\
\hline Nylon impregnated with urethane & 0.69 & 19.70 & 5.62 \\
\hline Urethane nylon & 0.69 & 0.0052 & 6.79 \\
\hline Neoprene coated nylon & 0.75 & 6.9 & 6.79 \\
\hline
\end{tabular}


International Journal of Mathematical, Engineering and Management Sciences

Vol. 4, No. 2, 420-431, 2019

https://dx.doi.org/10.33889/IJMEMS.2019.4.2-034

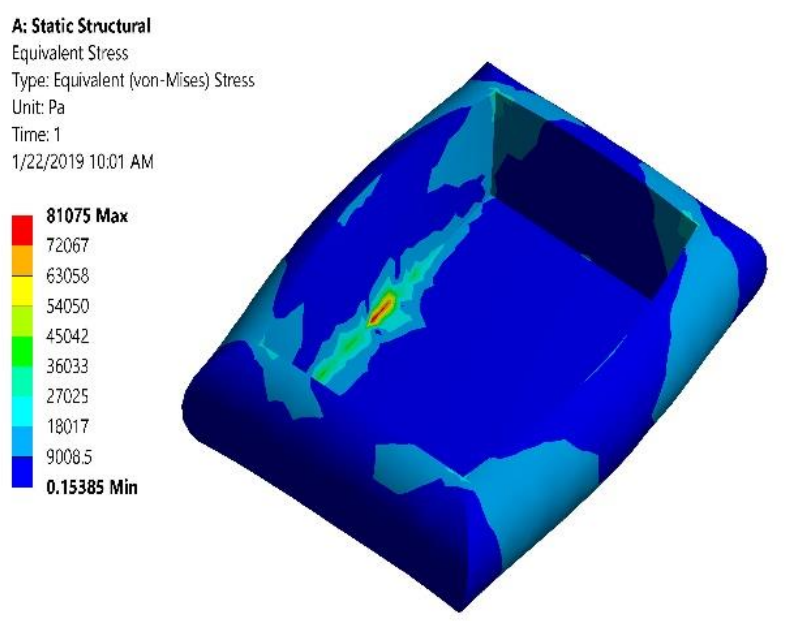

Figure 12. Stress contour of skirt

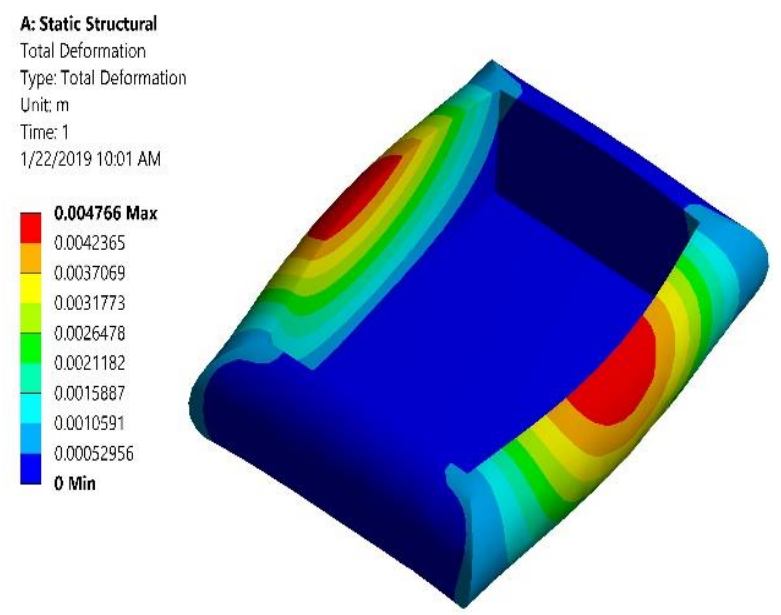

Figure 13. Deformation of skirt

FEA is carried out for various skirts materials and it is observed that (Table 2), the performance is quite similar for nylon family materials except for natural rubber. Stress contour and Deformation of the skirt are as shown in Figure 12 and Figure 13 respectively. Neoprene coated nylon is an allpurpose elastomer and an extremely versatile synthetic fabric capable of resisting degradation from sun, ozone and weather and performs well in contact with oils and chemicals. It is widely used in hovercraft skirts, industrial aprons, spray deck and adventure clothing.

\subsection{Integrated Structure}

After integrating UAV frame, hull, and skirt with the selected material and applying appropriate boundary conditions, FEA is carried out to evaluate the integrity and strength of the entire amphibious structure. Static analysis results indicated that, the designed structure experience very 
International Journal of Mathematical, Engineering and Management Sciences

Vol. 4, No. 2, 420-431, 2019

https://dx.doi.org/10.33889/IJMEMS.2019.4.2-034

minimal stress of $10.68 \mathrm{MPa}$ which is well within the allowable stress (Figure 14). A maximum deformation (Figure 15) of $1.71 \mathrm{~mm}$ can be seen at the four corners where the thrust from the propellers are acting due to cantilever structure.

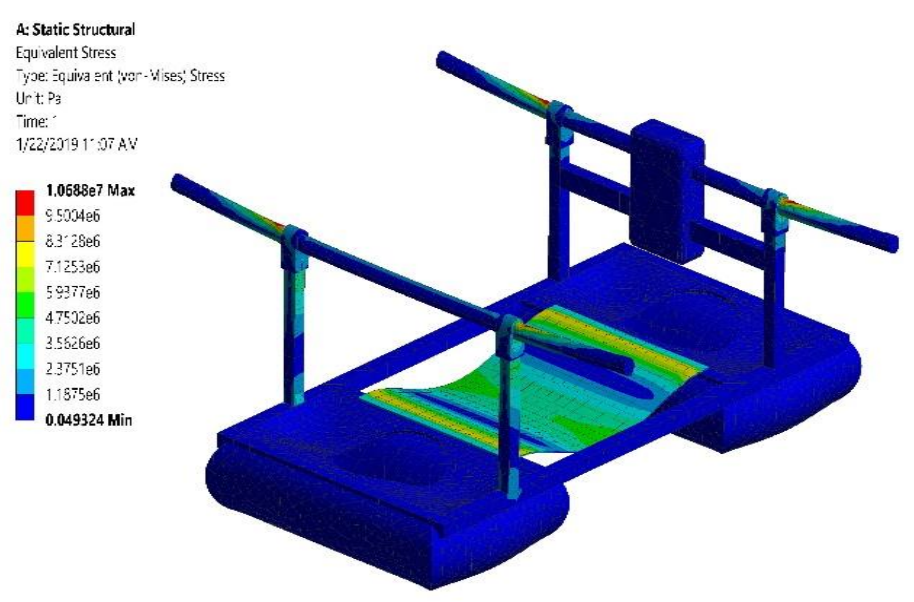

Figure 14. Stress contour of amphibious structure

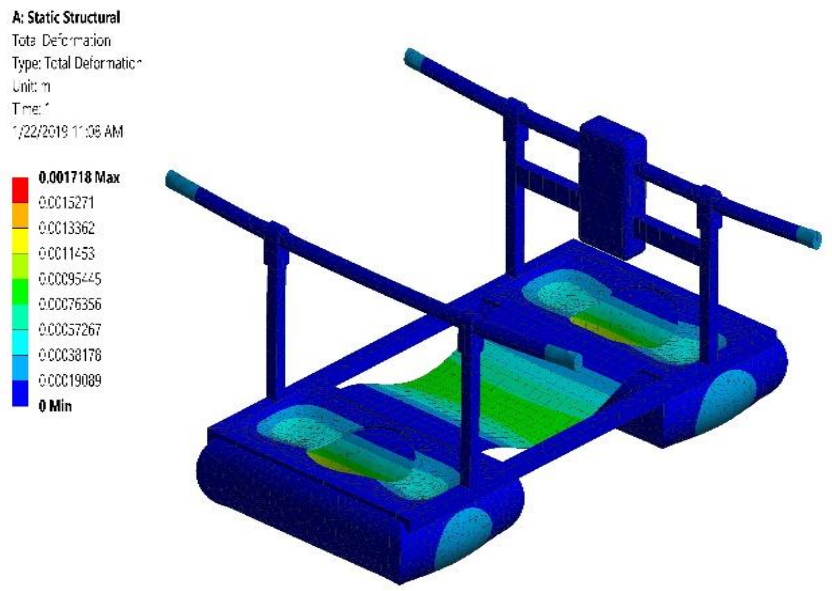

Figure 15. Deformation of amphibious structure

\section{Modal Analysis of Amphibious UAV}

The study vibration characteristics are considered to be an important aspect of all designs because, the mechanical systems have natural modes and may cause control disturbances. In order to determine the natural frequency of the amphibious structure, modal analysis is performed. When a specific load is applied to the mechanical system, the natural mode can be excited which can lead to catastrophic failure of the system. This leads to the importance of studying the resonance 
frequency of the UAV frame, which is the frequency that the system will be exited, to ensure that the natural modes will not be disturbed. The natural frequency of the amphibious model is computed and compared to the vortex shedding frequency of the propeller. These results are used to determine the physical operational speed limits for effective maneuvering. Identifying the sources of vibrations from the mechanical system is an important requirement to perform the modal analysis. In the case of an amphibian vehicle, the main source of mode excitation comes from the propulsion system (brushless electric motors and propellers). Since 30.5x9.7 (30.5-inch diameter, 9.7-inch pitch) propellers are chosen to lift the entire huge vehicle, and assuming standard sea-level air density, the vortex shedding frequency of the propellers can be deduced by using the information given for the motor rating $(\mathrm{KV})$ and supplied voltage $(\mathrm{V})$.

The Strouhal number of the propeller is estimated at 0.2 in our study. The dimensionless Strouhal number is used to determine the frequency induced by the propeller at different RPM which is given by,

$\mathrm{St}=\frac{f L}{U}$

St is the Strouhal number

$f$ is the vortex shedding frequency of the propeller

$U$ is the velocity of the flow through the propeller

$L$ is the characteristic length of the propeller

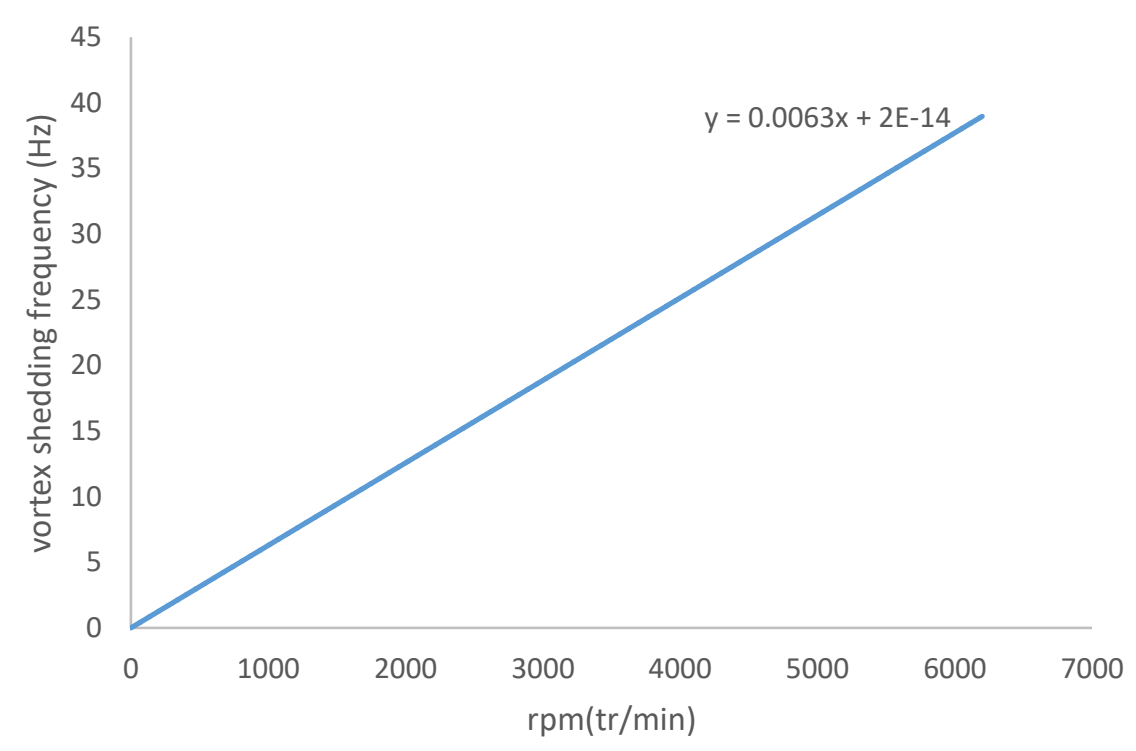

Figure 16. Linear correlation between vortex shedding frequency and motor RPM 
International Journal of Mathematical, Engineering and Management Sciences

Vol. 4, No. 2, 420-431, 2019

https://dx.doi.org/10.33889/IJMEMS.2019.4.2-034

A linear correlation between vortex shedding frequency and increase in motor RPM is observed in Figure 16. Meanwhile, the natural frequency of the aluminum frame is estimated, the first two modes are of bending of frames and then twisting is experienced. Other modes of vibration are occurred in the centre plate of the structure. Comparing the vortex shedding frequency with the natural frequency of the aluminum frame it can be determined that about $4200 \mathrm{rpm}$ can excite vibration severally. Modal analysis results predicted that, the first three natural frequencies of the frame are near to the vortex shedding frequency at higher speed of the motor. In order to keep the structure safe from resonance, the speed of the brushless motor has to be limited to below 4000 rpm.

\section{Conclusion}

A conceptual model of amphibious vehicle with integrating the features of multirotor and hovercraft systems is designed. Structural analysis is performed for various parts of the amphibious structure. FEA results for UAV frame suggested that carbon fibre and aluminium are obtained minimal stress and displacement. Aluminium is considered due to ease of availability and low cost. High density polyurethane foam in combination with wood experienced minimal displacement and selected for the hull. In nylon family, neoprene coated nylon is accounted as skirt material because of its excellent material characteristic with reference to its strength, flexing, twisting and elongation over a wide temperature range while having outstanding physical toughness. Frequency spectrum through modal analysis suggested that around an operating speed of $4000 \mathrm{rpm}$ resonance may occur and the amphibious UAV is to be propelled below and above this speed.

\section{Conflict of Interest}

The author confirms that there is no conflict of interest to declare for this publication.

\section{Acknowledgement}

Authors would like to thank the funding supported by DST-GITA (Ref: 2015RK0201103) and Centre for Autonomous System Research, Vel Tech Rangarajan Dr. Sagunthala R \& D Institute of Science and Technology, Avadi, Chennai for the technical support.

\section{References}

Amyot, J. R. (1989). Hovercraft technology, economics and applications (Vol. 11). Elsevier. ISBN: 9781483290850.

Collins, K. A. (1993). A concept of unmanned aerial vehicles in amphibious operations. Doctoral dissertation, Monterey, California. Naval Postgraduate School.

Detweiler, C., Griffin, B., \& Roehr, H. (2012, October). Omni-directional hovercraft design as a foundation for MAV education. In Intelligent Robots and Systems (IROS), 2012 IEEE/RSJ International Conference on (pp. 786-792). IEEE.

Frejek, M., \& Nokleby, S. (2008, May). Design of a small-scale autonomous amphibious vehicle. In Electrical and Computer Engineering, 2008. CCECE 2008. Canadian Conference on (pp. 000781000786). IEEE. 
International Journal of Mathematical, Engineering and Management Sciences

Vol. 4, No. 2, 420-431, 2019

https://dx.doi.org/10.33889/IJMEMS.2019.4.2-034

Gunwant, D. (2019). Stress concentration studies in flat plates with rectangular cut-outs using finite element method. International Journal of Mathematical, Engineering and Management Sciences, 4(1), 66-76.

Harkins, R., Ward, J., Vaidyanathan, R., Boxerbaum, A. X., \& Quinn, R. D. (2005, July). Design of an autonomous amphibious robot for surf zone operations: part II-hardware, control implementation and simulation. In Advanced Intelligent Mechatronics. Proceedings, 2005 IEEE/ASME International Conference on (pp. 1465-1470). IEEE.

Hasnan, K., \& Ab Wahab, A. (2012). First design and testing of an unmanned three-mode vehicle. International Journal on Advanced Science, Engineering and Information Technology, 2(1), 13-20.

Hassanalian, M., \& Abdelkefi, A. (2017). Classifications, applications, and design challenges of drones: a review. Progress in Aerospace Sciences, 91, 99-131.

Kumar, G. S., Painumgal, U. V., Kumar, M. C., \& Rajesh, K. H. V. (2018). Autonomous underwater vehicle for vision based tracking. Procedia Computer Science, 133, 169-180.

Liu, Z., Theilliol, D., Yang, L., He, Y., \& Han, J. (2018). Mode transition and fault tolerant control under rotor-tilt axle stuck fault of quad-TRUAV. IFAC-PapersOnLine, 51(24), 991-997.

Pisanich, G., \& Morris, S. (2002). Fielding an amphibious UAV: development, results, and lessons learned. In Digital Avionics Systems Conference, 2002. Proceedings. The 21st (Vol. 2, pp. 8C4-8C4). IEEE.

Solly, J., Früh, N., Saffarian, S., Aldinger, L., Margariti, G., \& Knippers, J. (2018, December). Structural design of a lattice composite cantilever. In Structures (in press) DOI: 10.1016/j.istruc.2018.11.019.

Valavanis, K. P., \& Vachtsevanos, G. J. (2015). Handbook of unmanned aerial vehicles. Springer Science+Business Media Dordrecht. Online ISBN 978-90-481-9707-1.

Vinayaga, K. K., Vasanthanathan, A., \& Nagaraj, P. (2018). Finite element modelling of smart piezoelectric beam using ANSYS®. Materials Today: Proceedings, 5(2), 7078-7085.

Vinayaga, K. K., Vasanthanathan, A., \& Nagaraj, P. (2018). Finite element modeling of smart piezoelectric beam using ANSYS®. Materials Today: Proceedings, 5(2), 7078-7085.

Yayla, M., Sarsilmaz, S. B., Mutlu, T., Cosgun, V., Kurtulus, B., Kurtulus, D. F., \& Tekinalp, O. (2013). Dynamic stability flight tests of remote sensing measurement capable amphibious unmanned aerial vehicle (A-UAV). AIAC, $7^{\text {th }}$ Ankara International Aerospace Conference, Ankara, Türkiye. 